\title{
A CRITERION FOR BIJECTIVITY OF MAPPINGS OF EUCLIDEAN SPACES
}

\author{
Albetã C. Mafra - Marcelo Tavares
}

\begin{abstract}
We study the following problem introduced by J. Hadamard in 1906: to find sufficient conditions for a local diffeomorphism of an Euclidean space to be a global diffeomorphism. J. Hadamard introduced a celebrated integral condition which is a sufficient condition for the bijectivity of a local diffeomorphism. In this paper we improve the classical result of Hadamard giving a new sufficient condition for a $C^{2}$ mapping to be bijective.
\end{abstract}

\section{Introduction}

In a remarkable paper [5] Hadamard begin to study the following problem: find sufficient conditions for a local diffeomorphism $F: \mathbb{R}^{n} \rightarrow \mathbb{R}^{n}$ to be a global diffeomorphism. In his work, Hadamard introduces an integral condition which is sufficient to assure the bijectivity of $F$. In [7], Lévy obtain a version of the Hadamard Theorem for Banach spaces. In [10], Plastock presents a proof of the Hadamard-Lévy theorem for Banach spaces using covering space theory. In this scenario, the Euclidean case is often stated as follows:

Theorem 1.1 (Hadamard-Plastock Theorem [5], [10]). Let $F: \mathbb{R}^{n} \rightarrow \mathbb{R}^{n}$ be a $C^{1}$ local diffeomorphism. If

$$
\int_{0}^{\infty} \inf _{\|z\|=r}\left\|D F(z)^{-1}\right\|^{-1} d r=\infty
$$

then $F$ is a diffeomorphism.

2010 Mathematics Subject Classification. 57R70, 57R35.

Key words and phrases. Global inversion of mappings, local diffeomorphism. 
In [8] an interesting generalization of the Hadamard-Plastock Theorem is obtained:

Theorem 1.2 (Nollet-Xavier Theorem [8]). Let $F: \mathbb{R}^{n} \rightarrow \mathbb{R}^{n}$ be a $C^{1}$ local diffeomorphism. If

$$
\int_{0}^{\infty} \inf _{\|z\|=r}\left\|D F(z)^{t} v\right\| d r=\infty, \quad \text { for all } v \in \mathbb{R}^{n} \backslash\{0\}
$$

then $F$ is a diffeomorphism.

For other recent results about invertibility of local diffeomorphisms see also [1], [3], [11], [12].

Now we start to describe our results. For $r \geq 0$ and $n \in \mathbb{N}$, denote

$$
\mathbb{S}_{r}=\mathbb{S}_{r}(n)=\left\{z \in \mathbb{R}^{n} ;\|z\|=r\right\} .
$$

Given a differentiable mapping $F: \mathbb{R}^{n} \rightarrow \mathbb{R}^{n}$, define the function

$$
\theta: \mathbb{R}^{n} \backslash F^{-1}(0) \rightarrow \mathbb{S}_{1}, \quad z \mapsto \frac{F(z)}{\|F(z)\|}
$$

For

$$
\mathfrak{S}=\left\{r \in[0, \infty) ; \mathbb{S}_{r} \cap F^{-1}(0) \neq \emptyset\right\},
$$

we can consider the function

$$
[0, \infty) \backslash \mathfrak{S} \rightarrow[0, \infty), \quad r \mapsto \inf _{\|z\|=r}\left\|D F(z)^{t} \theta(z)\right\| .
$$

Our main result is an improvement of Hadamard-Plastock Theorem in the $C^{2}$ case:

TheOrem 1.3. Let $F: \mathbb{R}^{n} \rightarrow \mathbb{R}^{n}$ be a $C^{2}$ mapping such that

(a) $F$ is a local homeomorphism;

(b) $D F(z)^{t} F(z) \neq 0$ whenever $F(z) \neq 0$.

If

$$
\int_{0}^{\infty} \inf _{\|z\|=r}\left\|D F(z)^{t} \theta(z)\right\| d r=\infty
$$

then $F$ is a bijective mapping.

In order to see that Theorem 1 improves Hadamard-Plastock Theorem, note that conditions (a) and (b) are satisfied whenever $F$ is a local diffeomorphism, that is, whenever $\operatorname{DF}(z)$ is non singular for all $z \in \mathbb{R}^{n}$. On the other hand, if $\mathbb{S}_{r} \cap F^{-1}(0)=\emptyset$, then

$$
\inf _{\|z\|=r}\left\|D F(z)^{-1}\right\|^{-1}=\inf _{\|z\|=r}\left(\inf _{\|v\|=1}\left\|D F(z)^{t} v\right\|\right) \leq \inf _{\|z\|=r}\left\|D F(z)^{t} \theta(z)\right\| .
$$


In the case $n=2$ we can weaken condition (a) in Theorem 1.3 and still obtain the properness of $F$ :

TheOREM 1.4. Let $F: \mathbb{R}^{2} \rightarrow \mathbb{R}^{2}$ be a $C^{2}$ mapping such that

(a) $F^{-1}(0)$ is discrete;

(b) $D F(z)^{t} F(z) \neq 0$ whenever $F(z) \neq 0$.

If

$$
\int_{0}^{\infty} \inf _{\|z\|=r}\left\|D F(z)^{t} \theta(z)\right\| d r=\infty
$$

then $F$ is a proper mapping.

We compare Theorems 1.3 and 1.4 to the Nollet-Xavier Theorem through a trivial example (cf. Example 3.5). Finally, we obtain a corollary due to Theorem 1.3 about perturbations of identity. The most classical version of the Perturbation of Identity Theorem asserts that if a $C$ mapping $\eta: \mathbb{R}^{n} \rightarrow \mathbb{R}^{n}$ satisfies $\|D \eta(z)\| \leq \lambda<1$ for all $z \in \mathbb{R}^{n}$, then the mapping $F(z)=z+\eta(z)$ is a diffeomorphism. We obtain the following:

Corollary 1.5 (Perturbation of Identity). Let $\eta: \mathbb{R}^{n} \rightarrow \mathbb{R}^{n}$ be a $C^{2}$ mapping. Suppose that, for some $r_{0}>0$,

$$
\frac{\left\|D \eta(z)^{t} \cdot(z+\eta(z))\right\|}{\|(z+\eta(z))\|} \leq \lambda<1,
$$

provided that $\|z\| \geq r_{0}$. Then the mapping $F(z)=z+\eta(z)$ is a diffeomorphism if, and only if, it is a local diffeomorphism.

The paper is organized as follows: In the second section we study the maximal interval of existence of the solutions of the vector field $X_{h}=\nabla h /\|\nabla h\|^{2}$, where $h: \mathbb{R}^{n} \rightarrow \mathbb{R}$ is a $C^{2}$ function. This is done from a compactness condition on $h$, which is a generalization of Palais-Smale condition. In Section 3 we prove the main theorems using the results from the previous section applied to the function $h(z)=\|F(z)\|^{2} / 2$.

\section{Global normal forms for submersions}

Let $h: \mathbb{R}^{n} \rightarrow \mathbb{R}$ be a $C^{2}$ function and denote by $\operatorname{Crit}(h)$ the set of the critical points of $h$. In the literature, the vector field

$$
X_{h}(z)=\nabla h(z) /\|\nabla h(z)\|^{2}, \quad z \in \mathbb{R}^{n} \backslash \operatorname{Crit}(h)
$$

is often used to study the levels of $h$. In fact, it is well-known that if $h$ is a submersion at every point and $X_{h}$ is complete (in particular, if $\|\nabla h\|$ is bounded away from zero), then $h$ is a globally trivial fibration and such trivialization is obtained from the (global) flow of $X_{h}$ (see [9], [8], [4]). The study of levels of a function is directly related to the problem of invertibility of mappings. In fact, 
the injectivity of a local diffeomorphism in the plane $F(x, y)=(f(x, y), g(x, y))$ follows from the connectedness of the levels of the submersions $f$ or $g$ (see [12]). This connectedness is achieved (in particular) by mean of the trivialization of such submersions. On the other hand, when we want to check the properness of a $C^{2}$ mapping $F: \mathbb{R}^{n} \rightarrow \mathbb{R}^{n}$ it is useful to study the vector field $X_{h}$ for $h=\|F\|^{2} / 2$, since the levels of $h$ are exactly the inverse-images of spheres under $F$. Indeed, we prove that the regular levels of $h$ are connected when the maximal interval of existence of the trajectories of $X_{h}$ is of the form $(\alpha, \infty)$ for an $\alpha$ that depends on the initial point and on the infimum of $h$ (cf. Lemma 3.1).

From the above discussion we conclude that it is useful to estimate the maximal interval of existence of the trajectories of $X_{h}$. The proposition below estimates such intervals from a condition of compactness on $h$, which is a generalization of the Palais-Smale condition ([9]).

Proposition 2.1. Let $h: \mathbb{R}^{n} \rightarrow \mathbb{R}$ be a $C^{2}$ mapping whose only possible critical value is its minimal value and denote $c=\inf h$. Suppose that there are a locally bounded function $\Psi:[0, \infty) \rightarrow[0, \infty)$ and a continuous function $T:(c, \infty) \rightarrow(0, \infty)$ such that

(a) $\Psi^{-1}(0)$ is discrete and $\Psi$ is continuous on $[0, \infty) \backslash \Psi^{-1}(0)$;

(b) $\int_{0}^{\infty} \Psi(r) d r=\infty$;

(c) $\|\nabla h(z)\| \geq \Psi(\|z\|)(T \circ h)(z)$, for all $z \in \mathbb{R}^{n} \backslash \operatorname{Crit}(h)$.

Then, for the orbit $\gamma$ of $X_{h}$ passing by $z \in \mathbb{R}^{n} \backslash \operatorname{Crit}(h)$, the maximal interval of existence is given by $(c-h(z),+\infty)$.

Proof. Let $z$ be a point in $\mathbb{R}^{n} \backslash \operatorname{Crit}(h)$. Write $c_{0}=h(z)$, and define

$$
H:\left(c-c_{0}, \infty\right) \rightarrow \mathbb{R}, \quad s \mapsto \int_{0}^{s} 1 / T\left(r+c_{0}\right) d r .
$$

Let $\gamma$ be a trajectory of $X_{h}$ by $z$ defined in its maximal interval of existence $\left(\omega_{-}, \omega_{+}\right)\left(\right.$see $[6$, p. 12] $)$. We have $\left(\omega_{-}, \omega_{+}\right) \subset\left(c-c_{0}, \infty\right)$, because

$$
h(\gamma(s))=s+c_{0} .
$$

Denote by $I$ the image of $\left(\omega_{-}, \omega_{+}\right)$by $H$, and let $s$ be the inverse of $H$ and suppose that $\omega_{+} \neq \infty$. Since the only possible critical points of $h$ are its minimal points, we have that

$$
\lim _{s \rightarrow \omega_{+}}\|\gamma(s)\|=\infty
$$

We divide the analysis into two cases:

Case 1. $\Psi^{-1}(0)$ is a finite set.

Denote $\Psi^{-1}(0)=\left\{r_{1}<\ldots<r_{k}\right\}$. By (2.3), there is a $t_{0} \in I$ such that if $s_{0}=s\left(t_{0}\right)$, then

$$
\left\|\gamma\left(s_{0}\right)\right\|>r_{k} .
$$


Define $\alpha(t)=(\gamma \circ s)\left(t+t_{0}\right)$, for $t \in\left[0, H\left(\omega_{+}\right)-t_{0}\right)$. We have that

$$
\alpha^{\prime}(t)=\frac{\nabla h(\alpha(t))}{\|\nabla h(\alpha(t))\|^{2}} \cdot(T \circ h)(\alpha(t)),
$$

thus,

$$
\left\|\alpha^{\prime}(t)\right\| \leq \frac{1}{\Psi(\|\alpha(t)\|)}, \quad \text { for all } t \in\left[0, H\left(\omega_{+}\right)-t_{0}\right) .
$$

Let $u$ be the maximal solution of the Cauchy problem

$$
\left\{\begin{array}{l}
u^{\prime}=\frac{1}{\Psi(u)}, \\
u(0)=\left\|\gamma\left(s_{0}\right)\right\| .
\end{array}\right.
$$

We claim that the maximal interval of existence of $u$ contains $[0, \infty)$. In fact,

$$
t=\int_{0}^{t} \Psi(u(\zeta)) u^{\prime}(\zeta) d \zeta=\int_{u(0)}^{u(t)} \Psi(r) d r .
$$

and the desired conclusion follows from (b). By (2.4), it follows from Lemmas 3.1, 3.2 and Theorem 4.1 of $[6$, p. 26] that

$$
\|\alpha(t)\| \leq u(t), \quad t \in\left[0, H\left(\omega_{+}\right)-t_{0}\right) .
$$

But

$$
\lim _{t \rightarrow H\left(\omega_{+}\right)-t_{0}}\|\alpha(t)\|=\lim _{s \rightarrow \omega_{+}}\|\gamma(s)\|=\infty .
$$

and this is a contradiction.

Case 2. $\Psi^{-1}(0)$ is an infinite set.

Denote $\Psi^{-1}(0)=\left\{r_{1}<\ldots<r_{k}<\ldots\right\}$. By (b) and since $\Psi$ is locally bounded, for each $k \geq 2$, we can choose a $r_{k-1}<l_{k}<r_{k}$, so that

$$
\sum_{k \geq 2} \int_{l_{k}}^{r_{k}} \Psi(r) d r=\infty .
$$

From (2.3), there is a $k_{0} \in \mathbb{N}$ such that, for each $k \geq k_{0}$, there is a $t_{k} \in\left[0, H\left(\omega_{+}\right)\right)$ so that

- $t_{k+1}>t_{k}$ and

- $\left\|\gamma\left(s\left(t_{k}\right)\right)\right\|=l_{k}$.

We claim that

$$
t_{k+1}-t_{k} \geq \int_{l_{k}}^{r_{k}} \Psi(r) d r .
$$

In fact, suppose that $t_{k+1}-t_{k}<\int_{l_{k}}^{r_{k}} \Psi(r) d r$, for some $k \geq k_{0}$. Consider $\alpha(t)=$ $\gamma\left(s\left(t+t_{k}\right)\right)$, for $t \in\left[0, t_{k+1}-t_{k}\right]$. We have

$$
\left\|\alpha^{\prime}(t)\right\| \leq \frac{1}{\Psi(\|\alpha(t)\|)}, \quad \text { for all } t \in\left[0, t_{k+1}-t_{k}\right]
$$


Let $u$ be the maximal solution of the Cauchy problem

$$
\left\{\begin{array}{l}
u^{\prime}=\frac{1}{\Psi(u)} \\
u(0)=l_{k}
\end{array}\right.
$$

There is a $\eta$ such that $\lim _{t \rightarrow \eta} u(t)=r_{k}$. But if $t \in[0, \eta)$, then

$$
t=\int_{0}^{t} \Psi(u(\zeta)) u^{\prime}(\zeta) d \zeta=\int_{l_{k}}^{u(t)} \Psi(r) d r
$$

Thus $\eta=\int_{l_{k}}^{r_{k}} \Psi(r) d r>t_{k+1}-t_{k}$. Applying Lemmas 3.1, 3.2 and Theorem 4.1 of $[6]$, we obtain

$$
\|\alpha(t)\| \leq u(t), \quad \text { for all } t \in\left[0, t_{k+1}-t_{k}\right] .
$$

But this is a contradiction, since $\left\|\alpha\left(t_{k+1}-t_{k}\right)\right\|=\left\|\gamma\left(s\left(t_{k+1}\right)\right)\right\|=l_{k+1}>r_{k}$. Hence we have (2.6). On the other hand,

$$
\infty>H\left(\omega_{+}\right)>\sum_{k \geq k_{0}}\left(t_{k+1}-t_{k}\right) \geq \sum_{k \geq k_{0}} \int_{l_{k}}^{r_{k}} \Psi(r) d r=\infty,
$$

and we also have a contradiction in the second case. Therefore, $\omega_{+}=\infty$.

Now, if $\lim _{s \rightarrow \omega_{-}} \gamma(s)$ consists of a critical point, then, by $(2.2), \omega_{-}=c-c_{0}$ since the only possible critical points of $h$ are minimal points. Suppose that $\omega_{-} \neq c-c_{0}$. In this case, we have $\lim _{s \rightarrow \omega_{-}}\|\gamma(s)\|=\infty$. Considering the curve

$$
\beta(t)=\alpha(-t)=\gamma(s(-t)), \quad t \in\left[0,-H\left(\omega_{-}\right)\right)
$$

and proceeding as above we obtain a contradiction and thus $\omega_{-}=c-c_{0}$.

If $F: \mathbb{R}^{n} \rightarrow \mathbb{R}^{n}$ is a $C^{1}$ mapping and $h=\|F\|^{2} / 2$, then the Proposition 2.1 is strongly related to the existence of a $F$-admissible flow (see Definition 3.1 and Theorem 4.1 of [11]). However, the existence of a $F$-admissible flow does not imply that $h^{-1}(0) \neq \emptyset$ (see Remark 3.1 of [11]). Below we prove that with a further condition on $T$ in Proposition 2.1 it is possible to ensure that $h$ assume its minimum.

Propostion 2.2. Let $h, \Psi$ and $T$ be as in the Proposition 2.1. If $c=\inf h \in$ $\mathbb{R}$ and

for some $\varepsilon>0$, then $h$ assumes its minimum.

$$
\int_{c}^{c+\varepsilon} \frac{1}{T(r)} d r<\infty
$$

Proof. Let $\gamma:\left(\omega_{-}, \omega_{+}\right) \rightarrow \mathbb{R}^{n}$ be an orbit of $X_{h}$ by $z \in \mathbb{R}^{n} \backslash \operatorname{Crit}(h)$, write $h(z)=c_{0}$ and define

$$
H:\left[c-c_{0}, \infty\right) \rightarrow \mathbb{R}, \quad s \mapsto \int_{0}^{s} 1 / T\left(r+c_{0}\right) d r .
$$


Let $\mathrm{s}$ be the inverse of $H$ and let $\beta$ be as in (2.8). We have that $-H\left(c-c_{0}\right) \in \mathbb{R}$ and, by the same arguments in the Proposition 2.1, if $\lim _{s \rightarrow c-c_{0}}\|\gamma(s)\|=\infty$, then we have a contradiction. Therefore, $\lim _{s \rightarrow c-c_{0}}\|\gamma(s)\|$ consists of a critical point, which is a minimal point of $h$.

A weak-Palais-Smale condition was defined in [13, Definition 3.4] in a very general context. Let $\varphi:[0, \infty) \rightarrow[0, \infty)$ be a continuous nondecreasing function such that

$$
\int_{0}^{\infty} \frac{1}{1+\varphi(r)} d r=\infty
$$

We say that a $C^{1}$ function $h: \mathbb{R}^{n} \rightarrow \mathbb{R}$ satisfies the weak-Palais-Smale condition if any sequence $\left\{z_{k}\right\}$ in $\mathbb{R}^{n}$ such that $h\left(z_{k}\right)$ is bounded and $\left\|\nabla h\left(z_{k}\right)\right\|(1+$ $\left.\varphi\left(\left\|z_{k}\right\|\right)\right) \rightarrow 0$ has a convergent subsequence. We say that $h$ satisfies the weakPalais-Smale condition at level $a \in \mathbb{R}$ if any sequence $\left\{z_{k}\right\}$ in $\mathbb{R}^{n}$ such that $h\left(z_{k}\right) \rightarrow a$ and $\left\|\nabla h\left(z_{k}\right)\right\|\left(1+\varphi\left(\left\|z_{k}\right\|\right)\right) \rightarrow 0$ has a convergent subsequence. For $\varphi(r) \equiv 0$, the weak-Palais-Smale condition is just the classical Palais-Smale condition.

If $h$ satisfies the conditions of Proposition 2.1, with $\Psi=1 /(1+\varphi(r))$, for some $\varphi$ continuous, nonnegative and nondecreasing, then $h$ is weak-Palais-Smale at each level $a \in(c, \infty)$. However, $h$ can satisfy the conditions of Proposition 2.2, without to be weak-Palais-Smale. Therefore, Proposition 2.2 assures the existence of a minimal point provided that $h$ is bounded from below and satisfies a (slightly different) kind of compactness (see Theorem 3.6 of [13]).

As mentioned above, the invertibility of a local diffeomorphism in the plane $F(x, y)=(f(x, y), g(x, y))$ is directly connected to the trivialization of the submersions $f$ or $g$ (see [12]). Therefore, it is interesting to study the case where $h$ is a submersion at every point of $\mathbb{R}^{n}$. This is done in the next two results:

Propostion 2.3. Let $h, \Psi$ and $T$ be as in Proposition 2.1. If $c=\inf h=$ $-\infty$, then $h$ gives a globally trivial fibration of $\mathbb{R}^{n}$ on $\mathbb{R}$ whose fibers are the levels of $h$.

Proof. This follows from the fact that $h$ is a submersion and $X_{h}$ defines a complete flow (see section 8 of [9]).

Given a $C^{1}$ submersion $h: \mathbb{R}^{n} \rightarrow \mathbb{R}$, we can consider the upper-semicontinuous function

$$
\Theta: \mathbb{R}^{n} \rightarrow[0, \infty), \quad z \mapsto \inf \left\{\|\nabla h(y)\| ; y \in h^{-1}(h(z))\right\} .
$$

By construction, $\Theta$ can be factored in the form $\Theta=T \circ h$, where $T$ is uppersemicontinuous and defined on the image of $h$. Since $\|\nabla h\| \geq \Theta=T \circ h$, it follows from the proposition below that $h$ is a globally trivial fibration provided that $1 / T$ is locally integrable (compare with [4]): 
Propostion 2.4. Let $h: \mathbb{R}^{n} \rightarrow \mathbb{R}$ be a submersion of class $C^{1}$ and $U=$ $h\left(\mathbb{R}^{n}\right)$. Suppose that there is an upper-semicontinuous function $T: U \rightarrow[0, \infty)$ such that

(a) $1 / T \in L_{\text {loc }}^{1}(U)$;

(b) $\|\nabla h\| \geq T \circ h$.

Then $h$ gives a globally trivial fibration of $\mathbb{R}^{n}$ on $U$ whose fibers are the levels of $h$.

Proof. Once again, let $\gamma:\left(\omega_{-}, \omega_{+}\right) \rightarrow \mathbb{R}^{n}$ be an orbit of $X_{h}$ by $z \in \mathbb{R}^{n}$, with $h(z)=c_{0}$, and write

$$
V=\left\{s ; s=h(x)-c_{0}, \text { for some } x \in \mathbb{R}^{n}\right\} .
$$

By section 8 of [9], it is enough to prove that $\gamma$ cuts each level of $h$. By $(2.2)$, it is enough to prove that $\left(\omega_{-}, \omega_{+}\right)=V$. We define

$$
H: V \rightarrow \mathbb{R}, \quad s \mapsto \int_{0}^{s} 1 / T\left(r+c_{0}\right) d r,
$$

and denote by $I$ the image of $\left(\omega_{-}, \omega_{+}\right)$by $H$. Since $T$ is upper-semicontinuous, $T$ assumes its maximum in each compact interval $J \subset V$. We write $\left.T\right|_{J} \leq C$. Therefore, if $a, b \in J$, we have

$$
|H(a)-H(b)| \geq \frac{1}{C}|b-a| .
$$

Let $s$ be the inverse of $H$. By (2.9), $s$ is Lipschitz on each compact interval and thus $s$ is absolutely continuous. Define $\alpha(t)=\gamma(s(t))$. Since $s$ is an absolutely continuous increasing function, each coordinate of $\alpha$ is absolutely continuous. Moreover,

$$
\alpha^{\prime}(t)=(T \circ h)(\alpha(t)) \cdot X_{h}(\alpha(t)),
$$

almost everywhere, and this implies that $\left\|\alpha^{\prime}(t)\right\| \leq 1$ almost everywhere. Therefore

$$
\|\alpha(t)-\alpha(0)\|=\left\|\int_{0}^{t} \alpha^{\prime}(\xi) d \xi\right\| \leq|t| .
$$

This implies that $I=\mathbb{R}$, so $\left(\omega_{-}, \omega_{+}\right)=V$, because $H$ is invertible.

\section{Proof of the main results}

In this section we prove the main theorems. For this we apply the results from the previous section to the function $h(z)=\|F(z)\|^{2} / 2$, where $F: \mathbb{R}^{n} \rightarrow \mathbb{R}^{n}$ is a $C^{2}$ mapping. The next lemmas are strongly related to some results in [11], just replacing the notion of $F$-admissible flow (see Definition 3.1 and Theorem 4.1 of [11]) by the local flow of $X_{h}$. These lemmas relate the properness of $F$ to the maximal interval of existence of the trajectories of $X_{h}$.

In that follows $F: \mathbb{R}^{n} \rightarrow \mathbb{R}^{n}$ is a $C^{2}$ mapping, $h(z)=\|F(z)\|^{2} / 2, X_{h}$ is as in $(2.1)$ and $c=\inf h$. 
Lemma 3.1. If $n \geq 2, F^{-1}(0)$ is discrete (possibly empty) and, for each $z \in \mathbb{R}^{n} \backslash \operatorname{Crit}(h)$, the maximal interval of existence of the trajectory of $X_{h}$ by $z$ is $(c-h(z),+\infty)$, then the regular levels of $h$ are connected.

Proof. Since $h$ is of class $C^{2}$, the vector field $X_{h}$ is of class $C^{1}$ and thus it defines a local flow which we denote by $\phi$. Let $c_{0}$ be a regular value in the image of $h$. It is easy to see that $\phi$ maps $h^{-1}\left(c_{0}\right) \times\left(c-c_{0}, \infty\right)$ homeomorphically on the set $\mathbb{R}^{n} \backslash F^{-1}(0)$, which is connected since $n \geq 2$.

Lemma 3.2. Let $F: \mathbb{R}^{n} \rightarrow \mathbb{R}^{n}$ be a $C^{2}$ local homeomorphism with $n \geq 2$. If $F^{-1}(0) \neq \emptyset$ and, for each $z \in \mathbb{R}^{n} \backslash \operatorname{Crit}(h)$, the maximal interval of existence of the trajectory of $X_{h}$ by $z$ is $(-h(z),+\infty)$, then $F$ is bijective.

Proof. Suppose that there is $z_{1} \neq z_{0}$ such that $F\left(z_{0}\right)=F\left(z_{1}\right)=0$. Since $F$ is a local homeomorphism, we can take disjoint open sets $U_{0} \ni z_{0}$ and $U_{1} \ni z_{1}$ which are sent homeomorphically by $F$ on an open ball $B \ni 0$. Let $c_{0}>0$ be a regular value of $h$ such that the ball $B_{\sqrt{2 c_{0}}}$ of radius $\sqrt{2 c_{0}}$ centered at the origin is contained in $B$, then we conclude that $h^{-1}\left(c_{0}\right)$ is not connected, which is an absurd by Lemma 3.1. Hence, $F^{-1}(0)=\left\{z_{0}\right\}$. Let $U_{0}, B$ and $c_{0}$ be as above and note that

$$
h^{-1}\left(\left[0, c_{0}\right]\right)=F^{-1}\left(B_{\sqrt{2 c_{0}}}\right) \subset U_{0},
$$

so $h^{-1}\left[0, c_{0}\right]$ is compact. Moreover, if $R$ is so that $R^{2} / 2>c_{0}$, then

$$
F^{-1}\left(\overline{B_{R}}\right)=h^{-1}\left(\left[0, c_{0}\right]\right) \cup h^{-1}\left(\left[c_{0}, R^{2} / 2\right]\right) .
$$

But if $\phi$ is the local flow of $X_{h}$, then domain of $\phi$ contains the set $h^{-1}\left(c_{0}\right) \times$ $\left[0,\left(R^{2} / 2\right)-c_{0}\right]$. Moreover,

$$
\phi\left(h^{-1}\left(c_{0}\right) \times\left[0,\left(R^{2} / 2\right)-c_{0}\right]\right)=h^{-1}\left(\left[c_{0}, R^{2} / 2\right]\right),
$$

then $h^{-1}\left(\left[c_{0}, R^{2} / 2\right]\right)$ is compact, $F^{-1}\left(\bar{B}_{R}\right)$ is compact and $F$ is proper. By a celebrated result in [2], $F$ is bijective.

In the case $n=2$ we have the following:

Lemma 3.3. Let $F: \mathbb{R}^{2} \rightarrow \mathbb{R}^{2}$ be a $C^{2}$ mapping. If $F^{-1}(0)$ is a non-empty discrete set and, for each $z \in \mathbb{R}^{n} \backslash \operatorname{Crit}(h)$, the maximal interval of existence of the trajectory of $X_{h}$ by $z$ is $(-h(z),+\infty)$, then $F^{-1}(0)$ consists of exactly one point and $F$ is proper.

Proof. Let $c_{0}>0$ be a regular value in the image of $h$. By Lemma 3.1, we have that $h^{-1}\left(c_{0}\right) \times\left(c-c_{0},+\infty\right)$ is homeomorphic to $\mathbb{R}^{2} \backslash F^{-1}(0)$ and thus $h^{-1}\left(c_{0}\right)$ is connected. Since $h^{-1}\left(c_{0}\right)$ has dimension 1 , it is diffeomorphic to $\mathbb{S}^{1}$ and $F^{-1}(0)$ contains exactly one point. Using the local flow $\phi$ of $X_{h}$ we conclude that $h^{-1}(d)$ is diffeomorphic to $\mathbb{S}^{1}$ for all $d>0$ and that $F$ is proper. 
The lemma below describes the connection between the hypothesis of the Theorem 1.3 and the maximal intervals of existence of $X_{h}$ :

LEMMA 3.4. If $F: \mathbb{R}^{n} \rightarrow \mathbb{R}^{n}$ is a $C^{2}$ mapping such that

(a) $F^{-1}(0)$ is discrete,

(b) $D F(z)^{t} F(z) \neq 0$ whenever $F(z) \neq 0$, and

(c) $\int_{0}^{\infty} \inf _{\|z\|=r}\left\|D F(z)^{t} \theta(z)\right\| d r=\infty$,

then $F^{-1}(0) \neq \emptyset$ and, for each $z \in \mathbb{R}^{n} \backslash \operatorname{Crit}(h)$, the maximal interval of existence of the trajectory of $X_{h}$ by $z$ is $(-h(z),+\infty)$.

Proof. If $z \notin F^{-1}(0)$, then

$$
\begin{aligned}
\|\nabla h(z)\| & =\left\|D F(z)^{t} F(z)\right\| \\
& =\left\|D F(z)^{t} \theta(z)\right\| \cdot\|F(z)\|=\left\|D F(z)^{t} \theta(z)\right\| \cdot \sqrt{2 h(z)} .
\end{aligned}
$$

Let $\mathfrak{S}$ be as in (1.2) and consider the functions

$$
\Psi:[0, \infty) \rightarrow[0, \infty), \quad r \mapsto \begin{cases}\inf _{\|z\|=r}\left\|D F(z)^{t} \theta(z)\right\| & \text { if } r \notin \mathfrak{S} ; \\ 0 & \text { if } r \in \mathfrak{S} .\end{cases}
$$

and

$$
T:(0, \infty) \rightarrow(0, \infty), \quad u \mapsto \sqrt{2 u} .
$$

By (b) we have that the only possible critical value of $h$ is its minimal value. By (3.1), if $z \in \mathbb{R}^{n} \backslash \operatorname{Crit}(h)$, then

$$
\|\nabla h(z)\| \geq \Psi(\|z\|)(T \circ h)(z) .
$$

Now observe that $\Psi$ is continuous in $[0, \infty) \backslash \Psi^{-1}(0)$. In fact, $\Psi$ is clearly upper semicontinuous in $[0, \infty) \backslash \mathfrak{S}$. Suppose that $\Psi$ is not lower semicontinuous at some $r_{0} \in[0, \infty) \backslash \mathfrak{S}$. In this case, there are $\varepsilon>0$ and a sequence $r_{k} \rightarrow r_{0}$ such that

$$
\Psi\left(r_{k}\right)<\Psi\left(r_{0}\right)-\varepsilon .
$$

Since $F^{-1}(0)$ is closed and discrete, we can suppose that $\mathbb{S}_{r_{k}} \cap F^{-1}(0)=\emptyset$ for all $k$. Therefore, there is a sequence $z_{k}$ such that $\left\|z_{k}\right\|=r_{k}$ and

$$
\Psi\left(r_{k}\right)=\left\|D F\left(z_{k}\right)^{t} \theta\left(z_{k}\right)\right\| \text {. }
$$

Taking subsequences, we can suppose that $z_{k}$ converges to some $z$ of norm $r_{0}$ and that $\theta\left(z_{k}\right)$ converges to $\theta(z)$. Hence, we have

$$
\Psi\left(r_{k}\right)=\left\|D F\left(z_{k}\right)^{t} \theta\left(z_{k}\right)\right\|<\Psi\left(r_{0}\right)-\varepsilon \leq\left\|D F(z)^{t} \theta(z)\right\|-\varepsilon,
$$

so

$$
\left\|D F(z)^{t} \theta(z)\right\|-\left\|D F\left(z_{k}\right)^{t} \theta\left(z_{k}\right)\right\|>\varepsilon
$$


which is absurd. On the other hand, $\Psi$ is locally bounded, since

$$
\Psi(r) \leq \sup _{\|z\|=r}\left\|D F(z)^{t}\right\| .
$$

By (3.2), the result follows from Propositions 2.1 and 2.2.

Now we prove the main theorems:

Proof of Theorem 1.3. For $n=1$ the result is straightforward. For $n \geq 2$, the proof follows from Lemmas 3.4 and 3.2.

Proof of TheOrem 1.4. The result follows from Lemmas 3.4 and 3.3.

The trivial example below is useful to compare our results with Nollet-Xavier Theorem:

ExAmple 3.5. Let $F: \mathbb{R}^{2} \rightarrow \mathbb{R}^{2}$ be the bijective $C^{\infty}$ mapping defined by

$$
F(z)=F(x, y)=\left(x^{3}, y\right) .
$$

Note that $F$ is not a local diffeomorphism, since $D F(z)$ is singular for $z=(0, y)$. However, we have

(1) $F^{-1}(0)=\{(0,0)\}$;

(2) $D F(z)^{t} F(z)=0$ if and only if $F(z)=0$.

Moreover, it is easy to verify that the integral condition (1.3) is satisfied. Therefore, this example is covered by Theorems 1.3 and 1.4. It is interesting to note that the integral condition (1.1) is not satisfied, since $\left\|D F(z)^{t} v\right\|=0$ for $z=(0, y)$ and $v=(1,0)$.

Proof of Corollary 1.5. If $z \notin F^{-1}(0)$ and $\|z\|>r_{0}$, then $\left\|D F(z)^{t} \theta(z)\right\|=\frac{\left\|\left(\operatorname{Id}+D \eta(z)^{t}\right)(z+\eta(z))\right\|}{\|z+\eta(z)\|} \geq 1-\frac{\left\|D \eta(z)^{t} \cdot(z+\eta(z))\right\|}{\|(z+\eta(z))\|} \geq 1-\lambda$.

The result follows from Theorem 1.3.

\section{REFERENCES}

[1] E.C. BAlreira, Incompressibility and global inversion, Topol. Methods Nonlinear Anal. 35 (2010), 69-76.

[2] S. BanACH And B. Mazur, Über mehrdeutige stetige abbildungen, Studia Math. 5 (1934), 174-178.

[3] M. Chamberland and G. Meisters, A Mountain Pass to the Jacobian conjecture, Canad. Math. Bull. 41 (1998), 442-451.

[4] F.G. Gascon And D. Peralta-Salas, On the construction of global coordinate systems in Euclidean spaces, Nonlinear Anal. 57 (2004), 723-742.

[5] J. Hadamard, Sur les transformations ponctuelles, Bull. Soc. Math. France 34 (1904), $71-84$.

[6] P. Hartman, Ordinary Differential Equations, Wiley, New York, 1964. 
[7] P. LÉvy, Sur les fonctions de lignes implicites, Bull. Soc. Math. France 48 (1920), 13-27. (in French)

[8] S. Nollet and F. Xavier, Global inversion via the Palais-Smale condition, Discrete Contin. Dyn. Syst. 8 (2002), 17-28.

[9] R.S. Palais, Morse theory on Hilbert manifolds, Topology 2 (1963), 299-340.

[10] R. Plastock, Homeomorphisms between Bannach spaces, Trans. Amer. Math. Soc. 200 (1974), 169-183.

[11] P.J. RABier, On global diffeomorphisms of Euclidean space, Nonlinear Anal. 21 (1993), 925-947.

[12] M. Sabatini, An extension to Hadamard global inverse function theorem in the plane, Nonlinear Anal. 20 (1993), 1069-1077.

[13] C.K. Zhong, A generalization of Ekeland's variational principle and application to the study of the relation between the weak P.S. condition and coercivity, Nonlinear Anal. 29 (1997), 1421-1431.

Albetã C. Mafra and Marcelo Tavares

Instituto de Matemática

Universidade Federal do Rio de Janeiro

P.O. Box 68530

21945-970 Rio de Janeiro, BRAZIL

E-mail address: albetan@im.ufrj.br, tavares@im.ufrj.br 